\title{
Micropapillary Carcinoma of the Bladder: Recent Advances
}

\author{
Jim $\mathrm{Hsu}^{1}$, Jae Y. Ro ${ }^{1,2}$
}

Cite this article: Hsu J, Ro JY: Micropapillary carcinoma of the bladder: recent advances. Ann Urol Oncol 2019;2(1): 26-35. https://doi. org/10.32948/auo.2019.07.01

\begin{abstract}
The 2016 WHO classification of tumors of the urothelial tract recently revised the classification of invasive urothelial carcinoma to include nested, microcystic, micropapillary, plasmacytoid, sarcomatoid, giant cell, and poorly differentiated variants, among others. In particular, invasive micropapillary carcinoma (IMPC) is now recognized as a distinct entity with aggressive features, including higher-stage disease, invasive features, and poorer response to intravesical chemotherapy. In this review, we highlight recent studies that further characterize the histopathology, immunohistochemistry, molecular mechanisms, and clinical implications of a diagnosis of IMPC. Because the correct morphologic diagnosis of IMPC is critical in terms of clinical management, we explore the diagnostic criteria of IMPC and differential diagnosis of urothelial IMPC from non-urothelial sites, highlighting studies that examine both traditional urothelial immunohistochemical markers as well as novel markers. We highlight recent advances in the molecular sub-categorization of IMPC, and review the differences compared to other forms of urothelial carcinoma. Optimal management of patients with IMPC is still unclear, although early cystectomy, regardless of pathologic stages, is recommended. We also highlight several studies that address the clinical challenges as well as current treatment protocols for IMPC.
\end{abstract}

Key words urothelial carcinoma, urinary bladder, micropapillary carcinoma, immunohistochemistry, molecular pathology

1. Department of Pathology and Genomic Medicine, Houston Methodist Hospital, Houston, 77030, USA.

2. Department of Pathology and Laboratory Medicine, Weill Cornell Medical College, New York, 10065, USA.

Correspondence: Jae Y. Ro (Department of Pathology and Genomic Medicine, Houston Methodist Hospital, 6565 Fannin Street, M227, Houston, TX 77030; Email: jaero@houstonmethodist.org). 
Introduction

Invasive micropapillary carcinoma (IMPC) of the bladder, an uncommon morphological variant of urothelial carcinoma, has been increasingly recognized as a distinct entity from conventional urothelial carcinoma since its first introduction by Amin et al. [1]. An aggressive form of urothelial carcinoma (Figure 1A-B), IMPC has been associated with aggressive clinicopathologic features, including lymphovascular invasion, lymph node metastasis, highstage disease, and poor response to intravesical chemotherapy (Figure 2A-B) [2-4]. These unfavorable prognostic features may also be seen with other forms of urothelial carcinoma, such as plasmacytoid [5, 6], sarcomatoid [7, 8], small cell [9], and other variants [10]; all of these should be considered during the workup of a bladder biopsy or cystectomy specimen, as these high-grade histological variants alter prognosis and management strategies.

The histological diagnosis and pathologic staging of IMPC dictates clinical management, and this has been supported by several outcome-based studies that have examined the standard of care, consisting primarily of radical cystectomy with or without (neo)adjuvant chemotherapy, and bladder-sparing therapies $[4,11]$. One of the first large-cohort studies to quantify the outcome of IMPC histology was a retrospective review of 100 patients seen at The University of Texas M. D. Anderson Cancer Center [3], which was comprised of predominantly male patients in the sixth decade with T1 to T2 disease. Ninety-four percent of patients presented with muscle-invasive disease, and $39 \%$ had died of the disease at a mean follow-up of 44 months. Crucially, patients in the study presenting with nonmuscle-invasive micropapillary carcinoma of the bladder had a survival rate no better than patients with invasive disease, and only $17 \%$ of patients who received intravesical therapy for nonmuscle-invasive disease were disease-free at 30 months of follow-up. Of 23 patients in the study who received neoadjuvant chemotherapy, pathologic downstaging occurred in 14 of 23 patients $(61 \%)$, while staging increased in $5(22 \%)$ patients; however, there was no significant benefit to overall survival (39.9 months) or 5-yr survival rate (49\%).

A subsequent study at Memorial Sloan-Kettering Cancer Center (MSKCC) prospectively reviewed 82 patients with non-metastatic IMPC ( $\geq$ cT2) in consideration of neoadjuvant therapy with four cycles of gemcitabine-cisplatin (12 weeks) [12]. Downstaging to pT0 occurred in 13 of $29(45 \%)$ patients that received neoadjuvant chemotherapy, compared to 2 of 15 (13\%) patients who did not. Furthermore, patients with pT0 disease had higher overall survival rates (25 vs. $92 \%)$ and lower rates of bladder cancer recurrence (21 vs. 79\%) at 24 months of follow-up. An additional study of 869 patients with IMPC and 389,603 patients with conventional urothelial carcinoma from the National Cancer Database (NCDB) [13] investigated three different surgical interventions - radical cystectomy, partial cystectomy, and transurethral resection of bladder tumor (the latter two denoted as bladder-preserving surgery). Of patients with cT1 disease who underwent bladder-

A

All cases of urothelial carcinoma

\section{Estimated New Cases in 2019: $\quad$ 80,470 $\%$ of All New Cancer Cases: $\underline{4.6 \%}$} Estimated Deaths in 2019:
\% of All Cancer Deaths: $\underline{17,670}$ $\underline{2.9 \%}$

\section{Overall 5-year survival}

\section{$77.1 \%$}

Survival, months after diagnosis
All 91.9 months
IMPC 44.7 months

$\mathrm{C}$

New cases per 100,000 persons

\begin{tabular}{|r|c|l|}
\hline \multicolumn{2}{|c|}{ Male } & \multicolumn{2}{|c|}{ Female } \\
\hline 35.2 & All races & 8.7 \\
\hline 38.5 & White & 9.5 \\
\hline 19.7 & Black & 6.6 \\
\hline 15.5 & $\begin{array}{c}\text { Asian/Pacific } \\
\text { Islander }\end{array}$ & 3.8 \\
\hline 14.9 & $\begin{array}{c}\text { American Indian/ } \\
\text { Alaska Native }\end{array}$ & $\mathbf{4 . 5}$ \\
\hline 19.5 & Hispanic & $\mathbf{5 . 1}$ \\
\hline 37.1 & Non-Hispanic & 9.2 \\
\hline
\end{tabular}

\section{Percent of new cases by age}

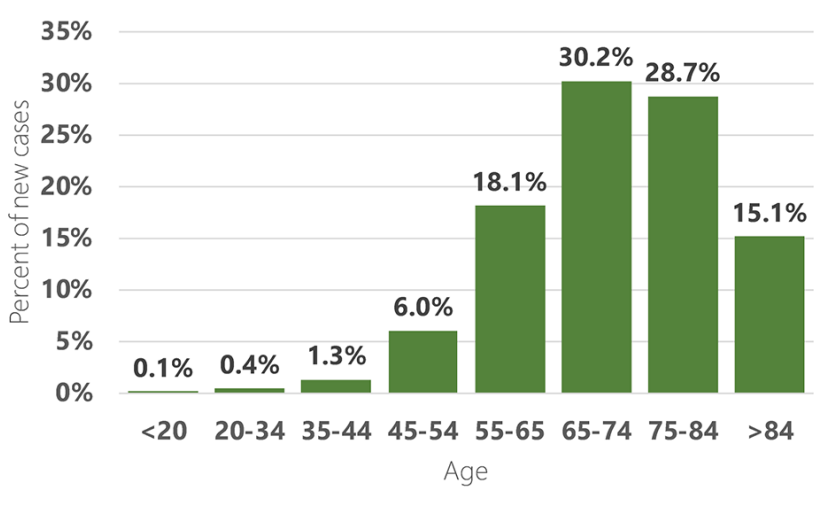

Figure 1. Epidemiology of urothelial carcinoma and the subset classified as noninvasive and muscle-invasive micropapillary carcinoma from the National Cancer Database (NCDB). A: Incidence and mortality, 2019, estimated; B: Overall survival; C: New cases per 100,000 persons in 2018; D: Percent of new cases diagnosed in 2018, by age. 
A

\section{Clinical Staging}

$$
\text { All }(n=389,603)
$$

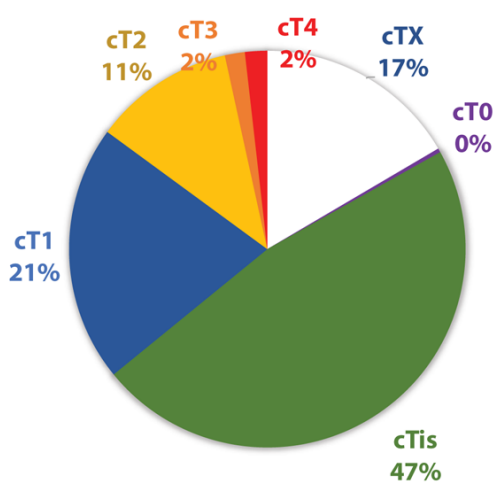

Micropapillary $(n=869)$

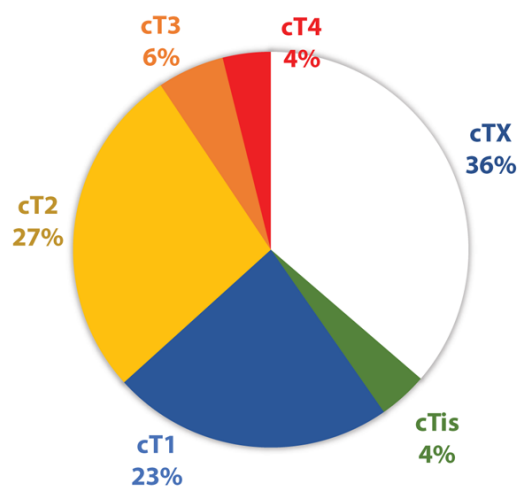

\section{Portion of node-positive} $\mathrm{N}(+)$ disease

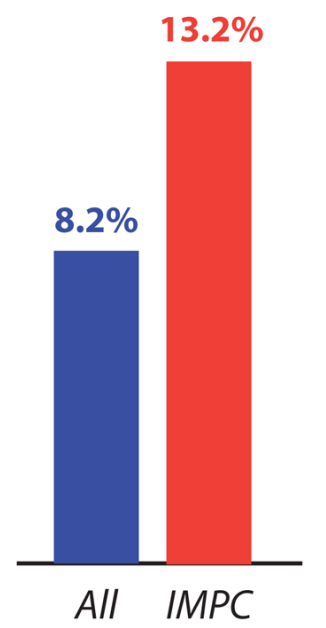

Figure 2. Clinical and pathologic staging of urothelial carcinoma and the subset classified as noninvasive and muscle-invasive micropapillary carcinoma from the National Cancer Database (NCDB). A: Clinically staged disease and proportion of node-positive disease (>N0); B: Pathologically staged disease and proportion of node-positive disease ( $>$ N0).

preserving surgery, the increase in median survival was not significant. A survival benefit of neoadjuvant chemotherapy prior to radical cystectomy was found for patients with conventional urothelial carcinoma; however, the benefits of neoadjuvant chemotherapy were abolished when restricted to the subset of patients with IMPC. Taken together, these three studies indicate that the definitive treatment for IMPC at all stages remains radical cystectomy, with a limited and controversial role for neoadjuvant chemotherapy (with $>$ cT2 disease) to potentially downstage disease prior to radical cystectomy.

\section{Epidemiology}

The National Cancer Institute (NCI) Surveillance, Epidemiology, and End Results (SEER) program have collated cancer incidence data since 1973, reflecting cancer incidences in diverse areas such as urban centers, suburbs, universities, and rural community hospitals. Approximately 80,000 individuals in the United States are diagnosed with cancer of the urinary bladder each year. Of these individuals, more than $75 \%$ of new cases are diagnosed in males [14]. Among patients in the SEER database, IMPC histology has only been identified in a total of 98 cases, for a frequency of approximately $0.01 \%$, and an age-adjusted incidence of 0.0139 per 100,000 patients [2]. The NCDB dataset supports a similarly low frequency, with 869 patients with IMPC and 389,603 patients with conventional urothelial carcinoma for an overall frequency of $0.2 \%$ of all urothelial carcinoma [13] (Figure 1A-D). In similar studies looking at patients diagnosed with urothelial carcinoma of all 

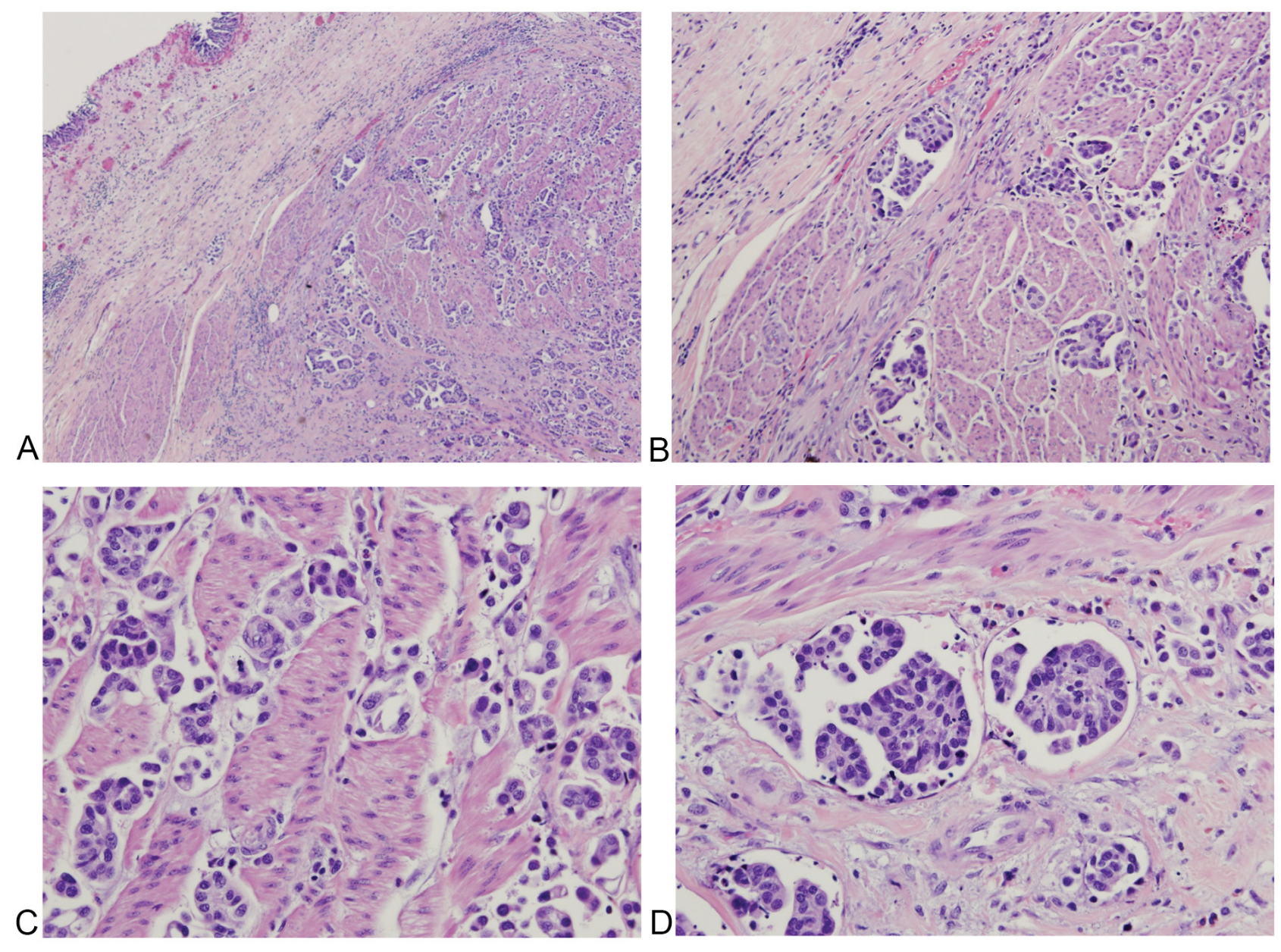

Figure 3. Histologic features of muscle-invasive micropapillary carcinoma of the bladder. A: H\&E, low magnification (40x), highlighting invasion of the muscularis propria; B: H\&E, intermediate magnification (200x); C-D: H\&E, high magnification (400x), highlighting sensitive, and specific morphologic features such as multiple nests in the same lacunar space, peripherally oriented nuclei, and back-to-back lacunae (Table 1).

subtypes, IMPC comprises approximately $0.2-8.2 \%$ of urothelial carcinomas $[15,16]$. Recognizing the scarcity of IMPC diagnosis, Wang et al. separately reviewed the literature and discovered six case studies with over ten cases each, published between 1980 and 2011, for a total of 213 cases of IMPC [2]. In summary, IMPC remains an uncommon to extremely rare variant of urothelial carcinoma. However, the increasing number of diagnosed cases of urothelial carcinoma, together with research on different histologic variants of urothelial carcinoma, may lead to increasing recognition of IMPC as a distinct histological variant of urothelial carcinoma.

\section{Pathology}

The first description of IMPC of the bladder is typically attributed to Amin et al., which describes a lesion with "slender, delicate filiform processes or tight papillary clusters reminiscent of papillary serous carcinoma of the ovary" [1]. However, the diagnosis of IMPC is frequently confounded with either micropapillary carcinoma from non-urothelial sites, or non-classical/'potential' cases of IMPC that may mimic other histologic forms of urothelial carcinoma. Sangoi et al. conducted a detailed study of 13 morphologic features that may be utilized to diagnose IMPC and quantified interobserver reproducibility of these features by using digital still images $(60 \mathrm{x}$ and 150x) of 30 separate cases of invasive urothelial carcinomas, including biopsies and resections of urothelial carcinoma from the urinary bladder or ureter, at Stanford University Medical Center [17]. These cases encompassed both classic IMPC ( $\mathrm{n}=$ 10) and urothelial carcinoma with stromal retraction that may potentially be diagnosed as IMPC $(n=20)$, and were reviewed by 14 genitourinary subspecialist pathologists. Overall interobserver agreement was moderate ( $\kappa: 0.54$ ) among the 14 pathologists, and the number of cases diagnosed as IMPC ranged from 9 of 30 (30\%) to 20 of $30(67 \%)$, with a median of 13 cases $(43 \%)$.

Among morphological features of classic IMPC, the presence of multiple nests in the same lacunar space has the highest sensitivity and specificity of all 13 features (Figure 3A-3B, Table 1), with intracytoplasmic vacuolization and epithelial ring forms also seen as highly specific (Figure 3C-3D, Table 1). In contrast, micropapillae are only moderately sensitive for classic IMPC, though still considered a sensitive and specific morphologic feature. While stromal retraction is a sensitive feature for IMPC (Table 2), Sangoi et al. have observed that invasive urothelial carcinoma with stromal retraction (Figure 4) is a much more common entity than the comparably rare IMPC, explaining the poor specificity of this diagnostic feature. Obviously, tumor nest width is an important criterion for the diagnosis of IMPC; however, conventional invasive urothelial carcinoma often display heterogeneous regions with small tumor nests (less than four cells across) admixed with larger tumor nests (more than 12 cells 
Table 1. Sensitive and specific morphological features for classic IMPC. Percentages denote sensitivity and specificity of feature under interobserver comparison, as described in Sangoi et al [17].

\begin{tabular}{lll}
\hline Feature & Sensitivity & Specificity \\
\hline Multiple nests in the same lacunar space & $90 \%$ & $95 \%$ \\
Intracytoplasmic vacuolization & $70 \%$ & $95 \%$ \\
Epithelial ring forms & $50 \%$ & $100 \%$ \\
Peripheral nuclei & $60 \%$ & $95 \%$ \\
Back-to-back lacunae & $80 \%$ & $85 \%$ \\
Micropapillae & $70 \%$ & $60 \%$ \\
\hline
\end{tabular}

across), which contributes to increased intra- and inter-observer variability and poor specificity for IMPC as a sole diagnostic criteria for this feature. The presence of randomly distributed nuclei and nest anastomosis are relatively insensitive and not specific diagnostic criteria. Similarly, medium-sized nests can be seen in all varieties of urothelial carcinoma, which are much more common in frequency than the micropapillary pattern. In addition, IMPC is almost always associated with lymphovascular invasion and lymph node metastasis with high $\mathrm{T}$ stage presentation.

\section{Immunohistochemistry}

Data on immunohistochemical markers for urothelial carcinoma is limited, although as these variants garner additional studies, more information has been obtained on traditional and novel urothelial marker expression. In a recent study, Paner et al. evaluated traditional and novel urothelial lineage markers on whole tissue sections from 130 cases of a spectrum of urothelial carcinoma and its variants [18]. Of urothelial carcinoma with or without divergent differentiation, overall positivity was observed as follows: GATA3 (50\%), S-100P (86\%), uroplakin III (20\%), thrombomodulin (40\%), cytokeratin 7 (CK7) (80\%), CK20 (55\%), p63 (87\%), and high molecular weight cytokeratin (HMCK) $(89 \%)$. Of micropapillary variants of urothelial carcinoma, of which there were 26 cases identified, overall positivity was observed as follows: GATA3 (86\%), S-100P (96\%), uroplakin III (38\%), thrombomodulin (38\%), cytokeratin 7 (CK7) (100\%), CK20 (73\%), p63 (54\%), and high molecular weight cytokeratin (HMCK) (96\%).

GATA binding protein 3 (GATA3) is a zinc finger transcription factor with important roles in T cell development and endothelial biology [19]. It is a commonly used marker of urothelial differentiation, and specifically sensitive for IMPC, with strong $3+$ staining in the majority of whole tissue sections. However, deficiencies have been reported on the use of GATA3 to identify urothelial carcinoma with squamous and glandular differentiation [20]. S100 demonstrates the highest sensitivity of all noncytokeratin markers for micropapillary carcinoma, but S100 is expressed in tumors of other origins, including gastric, esophageal, colorectal, liver, and lung carcinoma [21], thus limiting its utility for distinguishing micropapillary histology or urothelial versus non-urothelial carcinoma. Compared to conventional urothelial carcinoma, p63 positivity is less frequently seen (54\%), comparable to the plasmacytoid variant (50\%). A separate study of 20 cases of IMPC investigated the ability of various markers to distinguish IMPC from other histologic variants of urothelial carcinoma. The analysis showed that $\mathrm{p} 40$ expression is decreased in high-grade conventional urothelial carcinoma compared to lowgrade, and markedly decreased in IMPC [22]. However, GATA3,

Table 2. Other features associated with classic IMPC. Percentages denote sensitivity and specificity of feature under interobserver comparison, as described in Sangoi et al [17].

\begin{tabular}{lll}
\hline Feature & Sensitivity & Specificity \\
\hline Extensive retraction & $100 \%$ & $5 \%$ \\
Small nests (<4 cells across) & $100 \%$ & $5 \%$ \\
Columnar cells & $0 \%$ & $95 \%$ \\
Internal tufting & $0 \%$ & $90 \%$ \\
Marked nuclear pleomorphism & $10 \%$ & $75 \%$ \\
Nest anastomosis & $0 \%$ & $60 \%$ \\
Large nests $(>12$ cells) & $0 \%$ & $75 \%$ \\
Medium nests & $20 \%$ & $10 \%$ \\
Randomly distributed nuclei & $50 \%$ & $5 \%$ \\
\hline
\end{tabular}



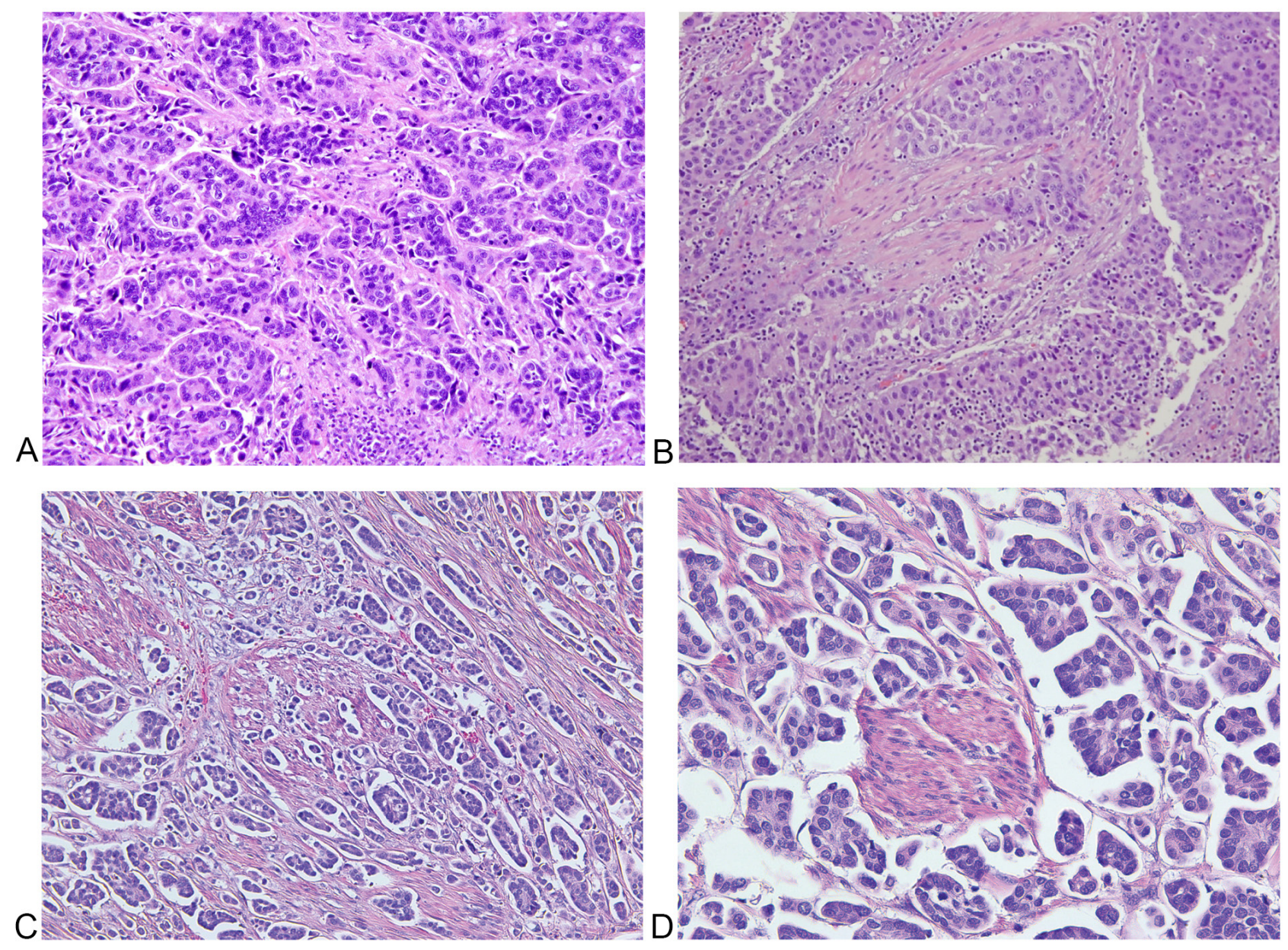

Figure 4. Features of conventional urothelial carcinoma with stromal retraction versus IMPC. A: Nests of tumor cells with stromal retraction at intermediate (200x) magnification, which may mimic small tumor nests; There is moderate nuclear pleomorphism with centrally oriented (non-inverted) nuclear polarization; B: Different section of the same case in (A), showing stromal retraction with more conventional urothelial carcinoma morphology; C: Intermediate (200x) and D: high (400x) magnification images of micropapillary histology for comparison, showing peripheral (inverted) polarization and multiple nests in the same lacunar space.

while decreased in high-grade conventional urothelial carcinoma, is largely retained in cases of IMPC.

Rather than focusing on staining intensity, other studies have investigated the role of the polarization of cell membrane proteins in distinguishing micropapillary histology from artifactual patterns that may mimic IMPC. Cell polarity reversal, defined by loss of apical-basal polarity, is characteristic of a transition from epithelial to migratory polarity, and is a hallmark of invasiveness [23]; it has been used in studies of breast [24] and gynecologic [25] tumors. A recent study by Hui et al. investigated combination staining with cell surface associated Mucin-1 (EMA), an apical epithelial marker, and E-cadherin in distinguishing micropapillary histology from stromal retraction artifacts that may mimic micropapillae [26] (Figure 4). Tumors with micropapillary histology show distinct membranous staining of EMA at the periphery, and E-cadherin that is negative on the periphery of tumor nests, characteristic of "inverted-polarization" (Figure 5). In contrast, urothelial carcinoma with retraction artifacts shows no distinct staining with EMA and E-cadherin at the periphery of tumor nests. Of tumors clinically classified as micropapillary, $89 \%$ show E-cadherin reversal, and $72 \%$ show membranous EMA staining, while only $10 \%$ of tumors classified as "retraction artifact" show either feature, suggesting that E-cadherin and EMA staining patterns may reliably distinguish IMPC from urothelial carcinoma with retraction artifact. An older study by Sangoi et al. evaluated the utility of MUC1, CA125, and Her2Neu to distinguish IMPC from invasive urothelial carcinomas with retraction artifact [27], and found that IMPC more often showed reactivity for these markers compared to conventional urothelial carcinoma, and that MUC1 reached statistical significance, with 23 of the 24 (96\%) IMPC and 15 of the $24(63 \%)$ invasive urothelial carcinomas (P $=0.0102)$ demonstrating reversal of MUC1 polarity staining. However, the specificity of MUC1 in this study was lower (37\%), limiting the discriminatory power to resolve cases of IMPC with retraction artifact compared to the study by Hui et al [26].

Given the histopathologic similarities of the micropapillary pattern among tumors from many different sites, including lung, breast, and ovaries, and the prognostic significance of the micropapillary pattern, it is often necessary to use immunohistochemistry to identify the origin of a metastatic IMPC. A study performed at Johns Hopkins collected 47 cases of IMPC from various sites (13 bladder, 6 lung, 16 breast, and 12 ovarian), with documented micropapillary pattern confirmed by inverted-polarization using MUC1 [28]. The most sensitive marker 

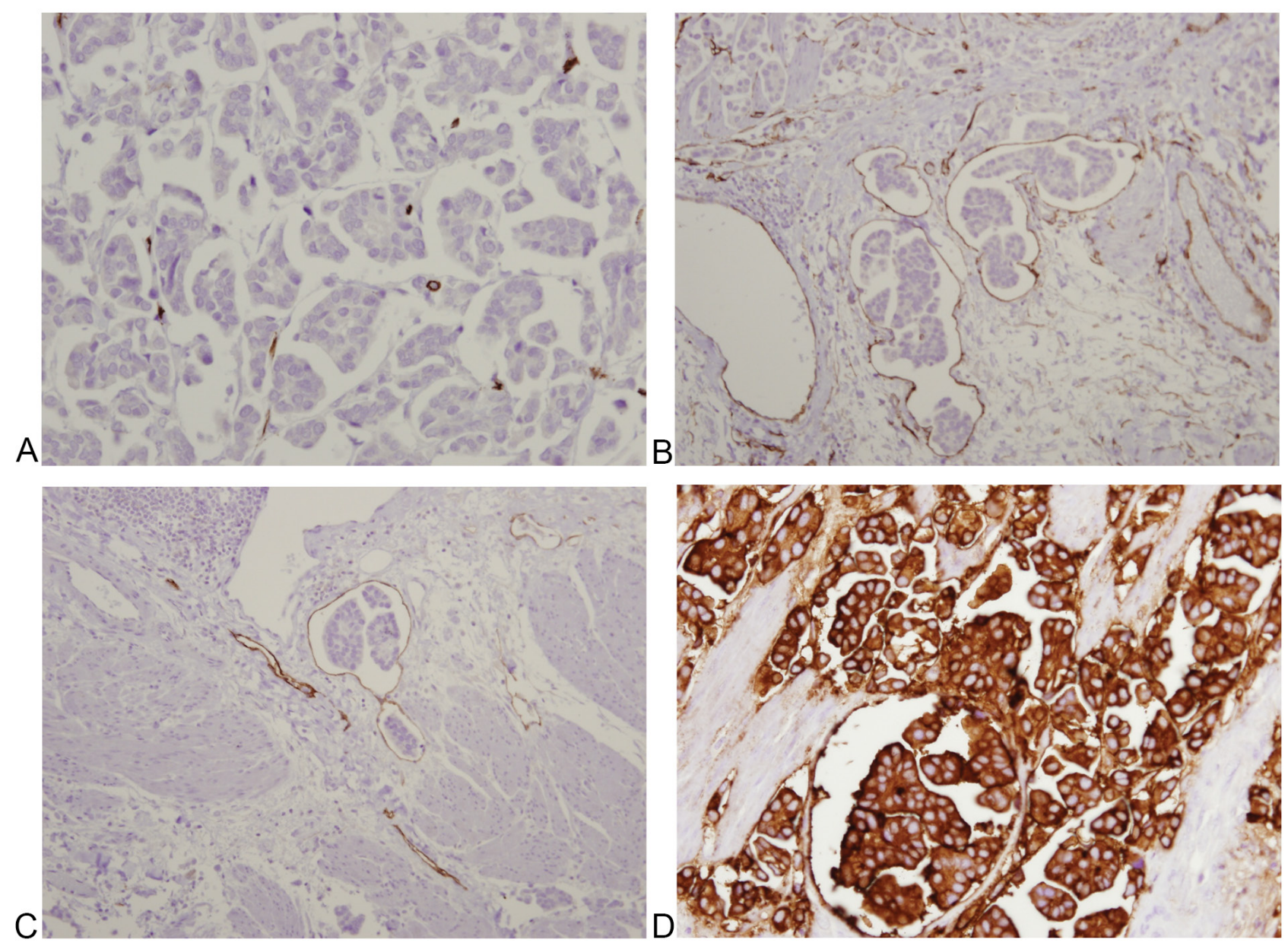

Figure 5. Immunohistochemical profile of muscle-invasive micropapillary carcinoma of the bladder highlighting inverted-polarization. A-B: CD34 demonstrates no staining of carcinoma cells, and staining of lacunae borders; C: D2-40 demonstrates staining of lacunae borders; D: EMA demonstrates membranous staining of tumor nests.

for specifically distinguishing urothelial IMPC from other invasive micropapillary carcinomas was uroplakin, with membranous and/ or cytoplasmic staining in $92 \%$ (11 of 12 ) of cases, and absent staining in all breast, lung, and ovarian micropapillary cancers tested. In contrast, CK20, while strongly labeling 54\% (7 of 13) of urothelial IMPC, also labeled 17\% (1 of 6) of lung cases, making it a less specific but still useful marker. TTF-1 is specific for lung micropapillary carcinoma, and was absent from all urothelial IMPC cases.

In summary, no immunohistochemical marker reliably distinguishes IMPC from other histologic subtypes of urothelial carcinoma; however, GATA3 retention and loss of p63 and p40 are characteristic, though not defining, features of this entity. Uroplakin positivity is a useful feature seen specifically in urothelial IMPC and absent from micropapillary cancers from other areas. Also, careful analysis of staining patterns in EMA/ E-cadherin labeled tumor for loss of cell polarity is another salient feature that may be useful for diagnosis.

\section{Molecular Alterations}

Interest in molecular alterations associated with invasive urothelial carcinoma, including IMPC, has been steadily increasing [29, 30]. Molecular studies of urothelial carcinoma have been translated into predicting chemotherapy response profiles via microarrays [31] and identification of specific therapeutic targets amenable to personalized therapy.

\section{HER2/ERBB2}

Human epidermal growth factor receptor-2 (HER2/ERBB2) is a transmembrane-bound receptor tyrosine kinase that is a wellknown oncogene implicated in many cancers, including breast, lung, ovarian, stomach, and uterine cancer [32]. Bladder cancer is typically not HER2-positive (only $6 \%$ to $8.7 \%$ ) [33, 34], but HER2 positivity is significantly higher for lymph node metastasis $(15.3 \%)$ [34]. Increased HER 2 expression has also been reported for IMPC through characterization by immunohistochemical and genomic studies. A study of Cleveland Clinic patients who underwent cystectomy between 1980 and 2008 demonstrated HER 2 protein expression positivity $(2+$ or $3+)$ in 13 of IMPC cases $(68 \%)$, and ERBB2 amplification (defined as a ratio $\geq 2.2$ ) of eight cases (42\%) with $100 \%$ concordance with immunohistochemical staining [35]. A case-control study of Mayo Clinic Cystectomy Registry patients with invasive urothelial carcinoma who underwent cystectomy from 1980 to 2008 showed that ERBB2 amplification was significantly associated with worse cancer-specific survival $(\mathrm{HR}=$ $4.3, p=0.0008)$ in patients with IMPC, compared to patients with 
non-amplified micropapillary carcinoma [11]. The 5-year cancerspecific survival of patients with IMPC with ERBB2 amplification was $0 \%$, compared to $40 \%$ for patients without ERBB2 amplification $(\mathrm{p}<0.001)$. Interestingly, ERBB2-amplified typical urothelial carcinoma was not associated with worse outcome compared to non-amplified typical carcinoma, demonstrating a potentially specific interaction between ERBB2 status and micropapillary histology, although only 9 of 100 patients in this case series displayed ERBB2 amplification of typical urothelial carcinoma. We note a recent whole-genome mRNA expression profiling study that demonstrates the association of ERBB2 positivity with luminal-type invasive bladder cancer, which displays distinct genomic signatures, transcriptional activation, and prognostic implications compared to basal-type or p53type bladder cancer [36]. The implications of this study for the micropapillary histologic subtype remain unclear.

\section{Gene Expression Profiles and Prognostic Indicators}

Continuing advances in molecular diagnoses have allowed for transcriptomics analyses to be extended into various families of tumors. Several studies have recently identified new molecular drivers of invasive bladder cancer [37], and a recent study extended the analysis to histologically diagnosed IMPC [38]. In this study, 128 high-grade muscle-invasive tumors from The Cancer Genome Atlas (TCGA) cohort, and 142 samples from M.D. Anderson Cancer Center, which included a subset of micropapillary carcinoma, were analyzed for gene expression analysis, tissue microarray, and immunohistochemistry of selected markers. The study discovered over 6,000 genes were differentially expressed in micropapillary carcinomas compared to conventional urothelial carcinoma, and highlighted multiple important oncogenic pathways converging on transformation, cell cycle regulation, DNA damage repair, and signal transduction. Hierarchical clustering revealed two well-defined clusters, labeled "A" and "B," containing almost exclusively conventional urothelial carcinomas and the majority of IMPC cases, respectively. Interestingly, the conventional tumors that segregated to cluster B contained at least some focal areas of micropapillary architecture.

When further analysis was done to examine the expression of luminal (e.g., KRT20, GATA3, uroplakins, ERBB2, etc.), basal (CD44, CDH3, KRT5, KRT6, and KRT14), and p53 markers, micropapillary cancers segregated almost exclusively into the luminal subtype, with almost half (45\%) demonstrating wildtype activated p53 signature, which corresponded to more aggressive clinical behavior. In contrast, the subset of conventional urothelial carcinoma segregated into luminal-type and basal-type carcinomas, similar to the segregation of invasive ductal carcinoma in the breast [39]. Further analysis identified a putative microRNA, miR-296, which showed consistent downregulation in IMPC, and concomitant overexpression of RuvB-like 1 (RUVBL1), an activator of the RAF/MEK/ERK pathway that has been shown to promote tumorigenesis in lung [40], breast [41], ovarian [42], and pancreatic cancer [43].

The significant variability of response to neoadjuvant chemotherapy and mixed results by several prospective and retrospective cohort studies $[12,44]$ has highlighted the need to predict chemosensitivity to identify potential responders and nonresponders in the general IMPC population. Kato et al. identified 33 cases of IMPC and constructed a panel of 26 predictive genes using quantitative reverse transcript PCR (RT-qPCR). They assessed i) chemosensitivity for a methotrexate, vinblastine, doxorubicin plus cisplatin (M-VAC) regimen, ii) a carboplatin plus gemcitabine $(\mathrm{CaG})$ regimen, iii) radiation therapy or iv) no chemotherapy, all prior to radical cystectomy [45]. Of patients who scored positive for M-VAC (indicating predicted responder),
6/7 demonstrated clinical response, and 16/18 of patients scoring positive for $\mathrm{CaG}$ also clinically responded, for an overall predictive accuracy (including negative non-responders to chemotherapy) of $37 / 40(92.5 \%)$ and response rate (of patients who received chemotherapy) of $88.0 \%(22 / 25)$. Furthermore, positive predicted responders demonstrated statistically improved survival to the negative predicted responder group $(\mathrm{p}=0.027)$. This study, while limited by small patient populations and a pre-determined clinical response threshold (tumor shrinkage of $60 \%$ ), nonetheless illustrates the potential of identifying potential chemosensitive responders and or targeted treatment through the application of molecular technologies.

\section{Conclusion}

The characterization, classification, immune-molecular profile, and optimal management of IMPC remains difficult, given the rarity of the diagnosis and unique histologic, immunohistochemical, and molecular profile of the tumor compared to conventional urothelial carcinoma. In terms of management, radical cystectomy remains the treatment of choice for all stages of disease, though the role of neoadjuvant or adjuvant chemotherapy remains controversial and is dependent on disease staging. The proper identification of the micropapillary histologic pattern is crucial, and practicing pathologists should note the considerable inter-observer variability associated with some classic micropapillary features (such as nest width) that are commonly used for diagnosis. While the specific molecular features and gene expression profiles of IMPC remain undetermined, it is clear that radical cystectomy remains the dominant treatment paradigm for any stage of disease, with ancillary roles for neoadjuvant chemotherapy for higher disease burden. Therefore, pathologists should be well versed in this increasingly recognized pattern of urothelial carcinoma.

\section{Acknowledgments}

The authors thank Sasha M. Pejerrey and Kathryn Stockbauer for their excellent editorial help.

\section{Funding}

None.

\section{Ethics approval and consent to participate}

This study did not require prior ethics approval or consent from human participants.

\section{Author contributions}

$\mathrm{JH}$ wrote the paper, reviewed references, and created the figures. JYR supervised and reviewed the paper, provided microscopic images for the figures, and is the corresponding author.

\section{Competing interests}

The authors declare no conflict of interest with the work.

\section{References}

1. Amin MB, Ro JY, el-Sharkawy T, Lee KM, Troncoso P, Silva EG, Ordóñez NG, Ayala AG: Micropapillary variant of transitional cell carcinoma of the urinary bladder. Histologic pattern resembling ovarian papillary serous carcinoma. Am J Surg Pathol 1994, 18(12): 1224-1232.

2. Wang J, Wang FW: The Natural History, Treatment Pattern, and 
Outcomes of Patients With Micropapillary Bladder Carcinoma. Am J Clin Oncol 2015, 38(5): 472-478.

3. Kamat AM, Dinney CPN, Gee JR, Grossman HB, Siefker-Radtke AO, Tamboli P, Detry MA, Robinson TL, Pisters LL: Micropapillary bladder cancer. Cancer 2007, 110(1): 62-67.

4. Kamat AM, Gee JR, Dinney CPN, Grossman HB, Swanson DA, Millikan RE, Detry MA, Robinson TL, Pisters LL: The Case for Early Cystectomy in the Treatment of Nonmuscle Invasive Micropapillary Bladder Carcinoma. J Urol 2006.

5. Keck B, Wach S, Stoehr R, Kunath F, Bertz S, Lehmann J, Stöckle M, Taubert H, Wullich B, Hartmann A: Plasmacytoid variant of bladder cancer defines patients with poor prognosis if treated with cystectomy and adjuvant cisplatin-based chemotherapy. BMC Cancer 2013, 13: 71.

6. Wang Z, Lu T, Du L, Hu Z, Zhuang Q, Li Y, Wang C-Y, Zhu H, Ye Z: Plasmacytoid urothelial carcinoma of the urinary bladder: a clinical pathological study and literature review. Int J Clin Exp Pathol 2012, 5(6): 601-608.

7. Fatima N, Canter DJ, Carthon BC, Kucuk O, Master VA, Nieh PT, Ogan K, Osunkoya AO: Sarcomatoid urothelial carcinoma of the bladder: a contemporary clinicopathologic analysis of 37 cases. Can J Urol 2015, 22(3): 7783-7787.

8. Bansal A, Kumar N, Sharma SC: Sarcomatoid variant of urothelial carcinoma of the urinary bladder. J Cancer Res Ther 2013, 9(4): 571-573.

9. Cheng L, Pan C-X, Yang XJ, Lopez-Beltran A, MacLennan GT, Lin H, Kuzel TM, Papavero V, Tretiakova M, Nigro K et al: Small cell carcinoma of the urinary bladder: a clinicopathologic analysis of 64 patients. Cancer 2004, 101(5): 957-962.

10. Urrea YR, Epstein JI: Sarcomatoid carcinoma associated with small cell carcinoma of the urinary bladder: a series of 28 cases. Hum Pathol 2017, 67: 169-175.

11. Schneider SA, Sukov WR, Frank I, Boorjian SA, Costello BA, Tarrell RF, Thapa P, Houston Thompson R, Tollefson MK, Jeffrey Karnes R et al: Outcome of patients with micropapillary urothelial carcinoma following radical cystectomy: ERBB2 (HER2) amplification identifies patients with poor outcome. Mod Pathol 2014, 27(5): 758-764.

12. Meeks JJ, Taylor JM, Matsushita K, Herr HW, Donat SM, Bochner BH, Dalbagni G: Pathological response to neoadjuvant chemotherapy for muscle-invasive micropapillary bladder cancer. BJU Int 2013, 111(8): E325-E330.

13. Sui W, Matulay JT, James MB, Onyeji IC, Theofanides MC, RoyChoudhury A, DeCastro GJ, Wenske S: Micropapillary Bladder Cancer: Insights from the National Cancer Database. Bladder Cancer 2(4): 415-423.

14. Siegel RL, Miller KD, Jemal A: Cancer Statistics, 2017. CA Cancer J Clin 2017, 67(1): 7-30.

15. Amin MB: Histological variants of urothelial carcinoma: diagnostic, therapeutic and prognostic implications. Mod Pathol 2009, 22(S2): S96-S118.

16. Alkibay T, Sözen S, Gürocak S, Işik Gönül I, Poyraz A, Ure I: Micropapillary pattern in urothelial carcinoma: a clinicopathological analysis. Urol Int 2009, 83(3): 300-305.

17. Sangoi AR, Beck AH, Amin MB, Cheng L, Epstein JI, Hansel DE, Iczkowski KA, Lopez-Beltran A, Oliva E, Paner GP et al: Interobserver reproducibility in the diagnosis of invasive micropapillary carcinoma of the urinary tract among urologic pathologists. Am J Surg Pathol 2010, 34(9): 1367-1376.

18. Paner GP, Annaiah C, Gulmann C, Rao P, Ro JY, Hansel DE, Shen SS, Lopez-Beltran A, Aron M, Luthringer DJ et al: Immunohistochemical evaluation of novel and traditional markers associated with urothelial differentiation in a spectrum of variants of urothelial carcinoma of the urinary bladder. Hum Pathol 2014, 45(7): 1473-1482.

19. Wilson BJ: Does GATA3 act in tissue-specific pathways? A meta- analysis-based approach. J Carcinog 2008, 7: 6.

20. Rao Q, Williamson SR, Lopez-Beltran A, Montironi R, Huang W, Eble JN, Grignon DJ, Koch MO, Idrees MT, Emerson RE et al: Distinguishing primary adenocarcinoma of the urinary bladder from secondary involvement by colorectal adenocarcinoma: extended immunohistochemical profiles emphasizing novel markers. Mod Pathol, 26(5): 725-732.

21. Higgins JPT, Kaygusuz G, Wang L, Montgomery K, Mason V, Zhu SX, Marinelli RJ, Presti JC, van de Rijn M, Brooks JD: Placental S100 (S100P) and GATA3: Markers for Transitional Epithelium and Urothelial Carcinoma Discovered by Complementary DNA Microarray. Am J Surg Pathol 2007, 31(5): 673-680.

22. Lin X, Zhu B, Villa C, Zhong M, Kundu S, Rohan SM, Yang $\mathrm{XJ}$ : The utility of $\mathrm{p} 63, \mathrm{p} 40$, and GATA-binding protein 3 immunohistochemistry in diagnosing micropapillary urothelial carcinoma. Hum Pathol 2014, 45(9): 1824-1829.

23. Gandalovičová A, Vomastek T, Rosel D, Brábek J: Cell polarity signaling in the plasticity of cancer cell invasiveness. Oncotarget 2016, 7(18): 25022-25049.

24. Gruel N, Benhamo V, Bhalshankar J, Popova T, Fréneaux P, Arnould L, Mariani O, Stern M-H, Raynal V, Sastre-Garau X et al: Polarity gene alterations in pure invasive micropapillary carcinomas of the breast. Breast Cancer Res 2014, 16(3): R46.

25. Hui Y, Ou JJ, Sung CJ, Lawrence WD, Quddus MR: Cell polarity reversal in ovarian low-grade serous carcinomas and micropapillary serous borderline tumours: immunohistochemical observations. Histopathology 2016, 69(5): 892-894.

26. Hui Y, Lombardo KA, Quddus MR, Matoso A: Cell Polarity Reversal Distinguishes True Micropapillary Growth From Retraction Artifact in Invasive Urothelial Carcinoma. Appl Immunohistochem Mol Morphol 2017: 1.

27. Sangoi AR, Higgins JP, Rouse RV, Schneider AG, McKenney JK: Immunohistochemical comparison of MUC1, CA125, and Her2Neu in invasive micropapillary carcinoma of the urinary tract and typical invasive urothelial carcinoma with retraction artifact. Mod Pathol 2009, 22(5): 660-667.

28. Lotan TL, Ye H, Melamed J, Wu X-R, Shih I-M, Epstein JI: Immunohistochemical panel to identify the primary site of invasive micropapillary carcinoma. Am J Surg Pathol 2009, 33(7): 1037-1041.

29. Glaser AP, Fantini D, Shilatifard A, Schaeffer EM, Meeks JJ: The evolving genomic landscape of urothelial carcinoma. Nature Rev Urol 2017, 14(4): 215-229.

30. Hurst CD, Knowles MA: Molecular Subtyping of Invasive Bladder Cancer: Time to Divide and Rule? Cancer Cell 2014, 25(2): 135-136.

31. Zhang QC, Petrey D, Deng L, Qiang L, Shi Y, Thu CA, Bisikirska B, Lefebvre C, Accili D, Hunter T et al: Structure-based prediction of protein-protein interactions on a genome-wide scale. Nature 2012, 490(7421): 556-60.

32. Moasser MM: The oncogene HER2: its signaling and transforming functions and its role in human cancer pathogenesis. Oncogene 2007, 26(45): 6469-6487.

33. Iyer G, Al-Ahmadie H, Schultz N, Hanrahan AJ, Ostrovnaya I, Balar $\mathrm{AV}$, Kim PH, Lin O, Weinhold N, Sander C et al: Prevalence and Co-Occurrence of Actionable Genomic Alterations in High-Grade Bladder Cancer. J Clin Oncol 2013, 31(25): 3133-3140.

34. Fleischmann A, Rotzer D, Seiler R, Studer UE, Thalmann GN: Her2 Amplification is Significantly More Frequent in Lymph Node Metastases From Urothelial Bladder Cancer Than in the Primary Tumours. Eur Urol 2011, 60(2): 350-357.

35. Ching CB, Amin MB, Tubbs RR, Elson P, Platt E, Dreicer R, Fergany A, Hansel DE: HER2 gene amplification occurs frequently in the micropapillary variant of urothelial carcinoma: analysis by dual-color in situ hybridization. Mod Pathol 2011, 24(8): 1111-1119.

36. Choi W, Porten S, Kim S, Willis D, Plimack ER, Hoffman-Censits J, Roth B, Cheng T, Tran M, Lee I-L et al: Identification of Distinct Basal and Luminal Subtypes of Muscle-Invasive Bladder Cancer 
with Different Sensitivities to Frontline Chemotherapy. Cancer Cell 2014, 25(2): 152-165.

37. Knowles MA, Hurst CD: Molecular biology of bladder cancer: new insights into pathogenesis and clinical diversity. Nature Rev Cancer 2015, 15(1): 25-41.

38. Guo CC, Dadhania V, Zhang L, Majewski T, Bondaruk J, Sykulski M, Wronowska W, Gambin A, Wang Y, Zhang S et al: Gene Expression Profile of the Clinically Aggressive Micropapillary Variant of Bladder Cancer. Eur Urol 2016, 70(4): 611-620.

39. Damrauer JS, Hoadley KA, Chism DD, Fan C, Tiganelli CJ, Wobker SE, Yeh JJ, Milowsky MI, Iyer G, Parker JS et al: Intrinsic subtypes of high-grade bladder cancer reflect the hallmarks of breast cancer biology. PNAS 2014, 111(8): 3110-3115.

40. Guo H, Zhang X-Y, Peng J, Huang Y, Yang Y, Liu Y, Guo X-X, Hao Q, An S, Xu T-R: RUVBL1, a novel C-RAF-binding protein, activates the RAF/MEK/ERK pathway to promote lung cancer tumorigenesis. Biochem Biophys Res Commun 2018, 498(4): 932 939.

41. Fan W, Xie J, Xia J, Zhang Y, Yang M, Wang H, Pan Y, Zhang M, Han B, Wu B et al: RUVBL1-ITFG1 interaction is required for collective invasion in breast cancer. Biochim Biophys Acta Gen Subj 2017, 1861(7): 1788-1800.

42. Li H, Tong X, Xu Y, Wang M, Dai H, Shi T, Sun M, Chen K, Cheng X, Wei Q: Functional genetic variants of RUVBL1 predict overall survival of Chinese patients with epithelial ovarian cancer. Carcinogenesis 2019.

43. Taniuchi K, Furihata M, Iwasaki S, Tanaka K, Shimizu T, Saito M, Saibara T: RUVBL1 directly binds actin filaments and induces formation of cell protrusions to promote pancreatic cancer cell invasion. Int J Oncol 2014, 44(6): 1945-1954.

44. Bertz S, Wach S, Taubert H, Merten R, Krause FS, Schick S, Ott OJ, Weigert E, Dworak O, Rödel C et al: Micropapillary morphology is an indicator of poor prognosis in patients with urothelial carcinoma treated with transurethral resection and radiochemotherapy. Virchow Arch 2016, 469(3): 339-344.

45. Kato Y, Zembutsu H, Takata R, Matsuura T, Kato R, Kanehira M, Iwasaki K, Yamada N, Katagiri T, Sugai T et al: A prospective study to examine the accuracies and efficacies of prediction systems for response to neoadjuvant chemotherapy for muscle invasive bladder cancer. Oncol Lett 2018, 16(5): 5775-5784. 